\title{
Simon au pays des soviets. Notes sur la réception de l'œuvre simonienne en Russie
}

\section{Alexey Vishnyakov}

\section{(2) OpenEdition}

1 Journals

Édition électronique

URL : http://journals.openedition.org/ccs/911

DOI : 10.4000/ccs.911

ISSN : 2558-782X

Éditeur :

Presses universitaires de Rennes, Association des lecteurs de Claude Simon

\section{Édition imprimée}

Date de publication : 31 mai 2014

Pagination : 145-168

ISBN : 9782753533387

ISSN : $1774-9425$

\section{Référence électronique}

Alexey Vishnyakov, «Simon au pays des soviets. Notes sur la réception de l'œuvre simonienne en Russie », Cahiers Claude Simon [En ligne], 9 | 2014, mis en ligne le 22 septembre 2017, consulté le 20 avril 2019. URL : http://journals.openedition.org/ccs/911 ; DOI : 10.4000/ccs.911 


\title{
SIMON AU PAYS DES SOVIETS Notes sur la réception de l'œuvre simonienne en Russie ${ }^{1}$
}

\author{
Alexey VISHNYAKOV \\ Institut d'État des Sciences humaines de la région de Moscou
}

Quel écrivain pourrait ignorer un pays avec un héritage littéraire si brillant (Gogol, Tourgueniev, Tolstoï et Dostoïevski)

et qui ne peut être nommé autrement que monumental? Et puis - comment ne pas s'intéresser à ce même pays devenu un polygone pour la doctrine (l'utopie?) marxiste $[\ldots]$,

le pays où sont apparues des personnalités aussi monumentales

(dans le mal comme dans le bien) que Lénine,

Trotski ou Staline ${ }^{2}$ ?

La Russie occupe dans l'ethnographie imaginaire de Simon l'une des premières places - après le motif espagnol et juste avant (ou juste après) le thème de la judaïcité. Le thème russe (et/ou soviétique) le hante durant presque toute sa vie, il est présent dans Le Tricheur (1945), La Corde raide (1947), Histoire (1967), Discours de Stockholm (1986), L'Invitation (1987), Album d'un amateur (1988), L'Acacia (1989), Le Jardin des Plantes (1997); il domine dans la nouvelle, Babel (1955), et apparaît comme une réminiscence et/ou un élément structural dans Gulliver (1952), Le Sacre du Printemps (1954), Le Vent. Tentative de restitution d'un retable baroque (1957). Et son dernier projet - une " cinquantaine de pages » qu'il déchire avant sa mort - c'est le nouveau retour

1. Je tiens à remercier la Fondation Maison des sciences de l'Homme (FMSH) pour l'aide généreuse qu'elle m’a apportée dans mon parcours de recherches depuis 2003.

2. Tiré des Réponses à quelques questions de Pavel Chinsky, juin 2001, publié dans Симон Клод, Приглашение - Москва, ОГИ, 2003, 135c. Simon Claude, L'Invitation (trad. en russe par Ekaterina Liamina, avec une réponse de Simon à quelques questions de Pavel Chinsky et une postface de Sergueï Zenkine), Moscou, OGI, 2003, p. 126. 
"sur son voyage en URSS, il a alors vingt-quatre ans, et sur la fulgurante passion pour Vera, la jeune femme d'Odessa ${ }^{3}$ ".

\section{LES TROIS VOYAGES DE CLAUDE SIMON EN RUSSIE}

Simon est venu en URSS trois fois et y a passé plus de quarante jours. La première fois, il a fait un voyage touristique en juin 1937. Le deuxième voyage a eu lieu dans la deuxième quinzaine de septembre 1984: Simon est arrivé en avion de Helsinki à Léningrad où il est descendu dans le célèbre "hôtel Astoria, ch. $\mathrm{n}^{\circ} 4$ " dont la fenêtre " ouvre directement sur la cathédrale Saint-Isaac " au centre de la ville, près du Palais d'Hiver dans lequel l'écrivain a vu soixante-sept ans après John Reed « une pièce [...] où les Gardes Rouges d'Octobre [...] ont signifié aux ministres réunis que pour eux les choses étaient terminées " (EI, p. 1110). À Moscou, Simon a eu une rencontre avec les lecteurs de la Bibliothèque d'État de la littérature étrangère. Ce voyage a été organisé par l'Union des écrivains soviétiques, juste après la publication de La Route des Flandres en russe en 1983. Le troisième voyage a duré une dizaine de jours et a lieu en octobre 1986, en réponse à l'invitation de participer au Forum d'Issyk-Koul.

Du point de vue de la connaissance du pays et de ses habitants, le premier voyage est le plus fructueux. Simon note toutes ses observations dans un carnet, il visite des musées (parmi d'autres - la Galerie Tretiakov, musée de l'art russe fondé par l'industriel Pavel Tretiakov ${ }^{4}$ ), des monuments, des usines, des kolkhozes, des églises, des colonies de pionniers et les « lieux les plus divers [...]. Plusieurs pages du carnet sont consacrées aux salaires, la comparaison avec le cours des prix, la durée du temps de travail ${ }^{5}$ ». Ce voyage, aussi bien que le troisième, a été plus d'une fois évoqué, décrit, réorchestré. Le deuxième voyage reste le plus inconnu, le plus secret même. Sa scène la plus émouvante, décrite dans Le Jardin des Plantes, évoque, dans le genre familier à Simon du portrait-histoire, une interprète léningradienne au "visage menu et grisâtre ", le chauffeur du taxi, ancien "capitaine dans l'artillerie " en " complet bleu, avachi, à la boutonnière duquel s'étalent en un petit éventail plusieurs rubans

3. Mireille Calle-Gruber, Claude Simon, Une vie à écrire, Paris, Le Seuil, coll. «Biographie », 2011, p. 436.

4. Personnage qui n'a rien de commun avec "Sergueï Tretiakoff, futuriste" (M. Calle-Gruber, op. cit., p. 97). Par ailleurs, Simon lui-même n'écrit pas correctement ce nom cher aux Russes dans le passage de $\mathrm{La}$ Corde Raide où il oppose à l'art traditionnel de l'Europe (dans lequel « une réalité artificielle " supplante "l'autre difficile et secrète, apparente et pourtant invisible») : "Il faut aller à Athènes au musée des Archaïques de l'Acropole, ou à la galerie Tétriakoff à Moscou, ou encore au Musée de l'Art Catalan à Barcelone si l'on veut entrer tout de suite dans le plaisir ", La Corde Raide, p. 12-13. À côté de sa peur devant les noms courants, Claude Simon avait une faiblesse presque julesvernienne pour les noms (propres ou toponymiques) exotiques, y compris russes qu'il n'écrivait pourtant pas toujours bien.

5. M. Calle-Gruber, op. cit., p. 96. 
de décorations » et l'aéroport qui cache «maintenant la ligne grisâtre des collines que les Allemands n'ont pas pu dépasser » (EI, p. 1111).

En parcourant l'Album d'un amateur ${ }^{6}$, on peut apprendre que Simon a encore visité, à Léningrad, la forteresse Pierre-et-Paul et le musée Dostoïevski. C'est pendant cette visite qu'a eu lieu une scène relative à trois problèmes brûlants représentée par Simon plus d'une fois avec des variations tantôt comiques (l'entretien qu'il a bien voulu m'accorder en témoigne) tantôt kafkaïennes (" une sorte d'interrogatoire " où l'écrivain aurait "choqué " ses " hôtes" ", interrogatoire qui en vérité ne pouvait être, selon moi, qu'une tentative de plaisanterie pour dissiper la méfiance du visiteur français $\left.{ }^{8}\right)$.

Le caractère et le contexte historique de ces trois voyages ne sont pas en effet ordinaires. De tous les intellectuels français invités en URSS, Simon est le seul à avoir essayé tous les modèles possibles de ce voyage (sauf, peut-être, le voyage sur invitation privée, quoique le dernier ait pu en avoir quelques traits) : en touriste individuel, en hôte de la structure officielle, en invité de marque au plus haut niveau; le moment de ces voyages est, lui aussi, loin d'être banal et effectué dans un contexte anodin: il s'agit, dans le premier cas, du dernier acte de la guerre de Staline contre les trotskistes, dans le second, de l'agonie d'un état désuet au bord du précipice, et dans le troisième, des tentatives désespérées de Gorbatchev de seconder une politique extérieure forte par des réformes intérieures efficaces.

Le degré d'implication de Simon dans la réalité soviétique a changé au cours de ces voyages. Si le premier est l'examen attentif, enthousiaste et ironique (un peu à la façon des héros des Lettres Persanes) d'un monde étrange et bariolé ("En Russie vous pouviez faire tout ce que vous vouliez si vous avez suffisamment d'argent. [...] vous offrir l'illusion d'être millionnaire [...]. Les Russes étaient très fiers de tout ce qu'il y avait dans leur pays, même si c'était pauvre, sale ou ridicule. C'était très sympathique et touchant, mais à la longue un peu agaçant ", $C R$, p. 14), couronné par un épisode sentimental paroxystique, le deuxième correspond à la dissection impitoyable des « illusions perdues » de la jeunesse snob, anarchiste et trotskiste, et le troisième au collage fantasmatique programmé par Simon d'avance, dans le style et l'optique de Swift et d'Orwell, et conduit du début à la fin selon cette technique d'une fantasmagorie dada.

6. Claude Simon, Album d'un amateur. Remagen-Rolandseck, Rommerskirschen, « Signatur », 1988.

7. Claude Simon, Lezione academica "Problèmes que posent le roman et l'écriture », Cahiers Claude Simon, $\mathrm{n}^{\circ} 7$, p. 225.

8. Pour plus de détails sur les circonstances du troisième voyage, voir mon article sur L'Invitation dans Dictionnaire Claude Simon, dir. M. Bertrand, préface de Dominique Viart, Champion, coll. "Dictionnaires et Références, 29 ", 2013, p. 538-543. 


\section{LES RÉTICENCES INITIALES DE LA CRITIQUE SPÉCIALISÉE}

Les traces laissées par les visites et les livres de Simon en URSS ne sont pas moins intéressantes, inattendues et même parfois extravagantes. Au premier regard, Simon n'a jamais été au centre de l'attention de la critique et du public soviétique, ce qu'on peut voir en comparant le nombre de ses traductions en russe, des articles qui lui ont été consacrés voire des simples mentions de son nom avec ceux d'A. Robbe-Grillet et de N. Sarraute'

En tout - et sans compter mon article qui accompagne la publication de notre entretien dans la revue Voprossy literatoury en 2003 et mes deux ouvrages sur la poétique de Simon dans le contexte de Nouveau Roman - j’ai trouvé quatorze sources susceptibles d'être évoquées ici, d'une grande variété de thèmes, de tonalités et de genres. Il est à noter que presque tous les auteurs de ces articles sont docteurs ès sciences, chercheurs et/ou traducteurs connus, spécialistes en langue et littérature française.

Le premier article consacré à la critique de la "nouvelle école » du roman est signé par Samariy Velikovski, traducteur, connaisseur de la littérature française, docteur ès sciences philosophiques. Sous le titre "Un fonctionnement à vide ${ }^{10}$ ", il est publié dans le magazine littéraire Inostrannaya literatoura (Littérature étrangère). Simon y est mentionné quatre fois comme « un représentant éminent de cette école » qui " construit son roman La Route des Flandres (1960), consacré à un épisode de la débâcle de l'armée française de l'été 1940 d'une manière telle qu'il est impossible d'y trouver quelque appréciation de la guerre parce que, selon lui, c'est le devoir des moralistes et des sociologues".

L'article suivant "Les peurs de Claude Simon » de Vladimir Ermilov ${ }^{11}$ en Literatournaya gazeta (Journal littéraire) est partagé entre la première et la quatrième pages. Il est assez grand (1 002 lignes ou 29000 signes), ce qui, ajouté à sa disposition en première page, montre l'importance de cette polémique

9. Par exemple, dans l'ouvrage académique Littérature française. 1945-1990, Moscou, "Naslédié", 1995 que je vais citer plus d'une fois, A. Robbe-Grillet est mentionné sur 73 pages, N. Sarraute, 56, M. Butor, 43 et C. Simon, 34, M. Duras, 27. Il est intéressant de noter que dans le chapitre consacré au Nouveau Roman de l'ouvrage de référence Littérature, XX' siècle, textes et documents, réunis par Bernard Lecherbonnier, Dominique Rincé, Pierre Brunel, Christiane Moatti, introduction historique de Pierre Miquel, Nathan, 2001 (p. 583-610), le palmarès des mentions est à peu près le même: A. RobbeGrillet est mentionné 49 fois, N. Sarraute, 17, M. Butor, 30, C. Simon, 18 et M. Duras, 10.

10. Samariy Velikovski, Un fonctionnement à vide, "Littérature étrangère », $\mathrm{n}^{\circ} 1,1963$, p. 176-191 (et Les illustrations littéraires: les fragments des romans de N. Sarraute (Le Planétarium), d'A. Robbe-Grillet (Dans le labyrinthe) et de M. Butor (Degrés), p. 191-206). L'article est très important, certes, mais ces trois « illustrations " ont eu encore plus d'importance, étant les premiers textes du Nouveau Roman accessibles au lecteur soviétique. Il faut préciser tout de même que pratiquement tous leurs livres se trouvaient dans les bibliothèques centrales de Moscou et de Léningrad. Par exemple, des livres de Simon, seuls Le Tricheur et Triptyque étaient absents des deux métropoles, et j'ai reçu Le Sacre du printemps de Léningrad.

11. Vladimir Ermilov, « Les peurs de Claude Simon ", Journal littéraire, 7 septembre de 1963, n 108, p. 1, 4. 
(c'est le sous-titre de l'article) aux yeux de la rédaction de ce journal principal des écrivains soviétiques. Non sans satisfaction, on peut constater que l'article d'un certain M. Kouznetsov (avec le même sous-titre polémique), La politique "poursuivie " par Robbe-Grillet (22 octobre, $\mathrm{n}^{\circ} 127$ ), est deux fois moins grand (470 lignes) et se trouve à la page 4 parmi les articles consacrés aux nouvelles culturelles internationales, ce qui signifie que la rédaction accorde une plus grande importance à la discussion avec Simon qu'au débat avec le Pape du Nouveau Roman!

L'article de Ermilov, aussi bien que celui de Simon «Et si les écrivains révolutionnaires jouaient le rôle de la presse du cœur? " paru dans L'Express le 25 juillet 1963, est l'écho d'une discussion au Séminaire des écrivains de l'Est et de l'Ouest en Finlande, l'été 1963. L'interprète finnois Reijo Nikkilä écrit: "En été 1963, le premier séminaire a été organisé. Le séminaire devait servir de pont pour les écrivains, critiques et traducteurs de l'Est (l'URSS) et de l'Occident (les pays capitalistes). Le thème de 1963 était L'écrivain et les préjugés. [...] Les thèmes indiquent seulement la direction, les discussions ont toujours débordé leur cadre. Par exemple, l'événement principal du premier séminaire a été une bataille orale entre Vladimir Ermilov (contemporain de Maïakovski et l'un de ses bourreaux) et l'écrivain français Claude Simon sur la question de la primauté à accorder au réalisme socialiste ou au modernisme. Pendant longtemps encore, ils ont continué ce débat amorcé dans le séminaire sur les pages de la Literatournaïa Gazeta et du Figaro littéraire " (hebdomadaire finnois édité en russe Спектр [Éventail], n 25, 21/06/2007).

La manière typique de mener la discussion avec l'Occident qui régnait dans le journalisme soviétique des années 30-70 se basait sur les accusations et les commentaires moqueurs ou grossiers de brèves et rares citations arrachés à leur contexte. Le plus souvent, la position opposée était représentée sous la forme d'une caricature déformée. De ce point de vue aussi, l'article de Ermilov malgré tout son pathos critique est frappant pour celui qui connaît les mœurs d'autrefois. Les 169 lignes (soit 17 \%) de l'article de ce critique reproduisent sous forme de citations juxtaposées ou en substance le texte de l'article de Simon. Cela montre tout le sérieux des intentions de l'un des théoriciens littéraires soviétiques les plus connus et les plus sévères ${ }^{12}$ des années 1920-1960. Tout l'article est divisé en chapitres sur les peurs de Claude Simon qui sont en nombre de 5: Peur 1: devant la science, Peur 2: devant la recherche de la vérité

12. Le nom de Ermilov est pour toujours lié à celui de Mä̈akovski qu'il critiquait sans pitié et le poète révolutionnaire regrettait dans sa lettre d'adieu de ne pas « mener au bout l'engueulade » avec lui. Ici on peut se rappeler encore une anecdote sur Ermilov des années 60. Sur l'écriteau attaché au portail de sa datcha où il était écrit: Attention au chien méchant, quelque voisin des milieux littéraires aurait ajouté et sans scrupules. On raconte, d'ailleurs, la même histoire sur le bourreau de la génétique soviétique Trofime Lyssenko. 
(la plus grande partie de l'article), Peur 3: devant la politique, Peur 4: devant l'humanité ou l'inhumanité? Peur 5: pour l'avenir de l'art. Ermilov cite les noms de Lénine, de Flaubert et de Madame Bovary, d'Aristote, de Pouchkine et de sa Tatiana, de Tolstö̈ et de Wronski de Anna Karénine, de Dostoïevski, Tchekhov, Shakespeare, Stendhal, Balzac.

Il est assez difficile de résumer toutes les idées de cet article majeur pour la perception de l'œuvre simonienne en URSS. D'abord, son ton est assez bienveillant (il est dit par exemple: "L'auteur réfléchit à des choses très importantes, c'est pourquoi soyons très attentifs envers ce que Claude Simon dit »), ce qui est relativement rare dans les articles de ce type et de cette époque. Le critique soviétique n'est pas choqué ou indigné par les propos de l'article de Simon, il feint d'en être étonné et même parfois amusé.

Les réflexions de l'écrivain français provoquent avant tout un sentiment d'étonnement. Dans la critique soviétique, avaient déjà lieu dans les années 20-30 les discussions sur l'impossibilité et l'inadmissibilité du refus de distinction entre l'art et la science. [...] Et malgré ses bonnes intentions éventuelles, c'est ici que se cachent les fondements des réflexions de Simon. L'art a ses propres buts, ses méthodes, sa façon particulière de comprendre la réalité et l'homme. Et c'est assez étrange d'être obligé de redire ces vérités bien connues.

Ermilov se croit obligé de donner des leçons à Simon, ce à quoi il était habitué vu sa position au sein de la littérature soviétique de l'époque.

Est-ce que la littérature est capable de mettre en doute les structures sociales si elle n'a pas le droit de faire la politique? Quel imbroglio! Il apparaît toujours avec la prémisse incorrecte. Il faut encore ajouter qu'à l'époque de l'arme nucléaire et de la menace de la guerre atomique, l'appel aux écrivains [fait par Claude Simon] de s'éloigner de la politique a une très concrète signification politique objective. Il faut croire que Claude Simon ne le croit pas, autrement, il n'aurait pas pris part au congrès international, L'écrivain et la coexistence pacifique. Mais est-ce qu'il ne doit pas réfléchir à la signification objective de sa demande aux romanciers de ne pas faire de la politique? [...] Peut-être, Claude Simon doit-il réfléchir au fait que la peur devant l'homme, devant une personnalité humaine avec tout son caractère unique, l'abstention de l'affirmation de sa valeur n'est pas si loin de la capitulation devant l'inhumanité? Et de nouveau je souligne qu'il ne s'agit pas des aspirations subjectives de Claude Simon mais de leur sens objectif.

Dans plusieurs endroits de l'article, on peut sentir de la sympathie éprouvée par le rival des formalistes et de Maïakovski, envers Simon qui se cache derrière des remontrances, comme, par exemple, dans cette chute de l'article:

Probablement, Claude Simon appartient aux écrivains qui sont tellement terrorisés par le progrès actuel de la science qu'ils sont capables d'oublier que l'art a sa propre forteresse inébranlable. [...] Encore et encore, nous nous heurtons dans les thèses de Claude Simon aux idées qu'on ne peut qualifier que comme préhistoriques. D'un côté, Claude Simon affirme que tout change, rien ne se répète et que ce qui a été vrai hier ne l'est pas aujourd'hui. Mais d'un autre côté, c'est lui-même qui répète les idées sur l'art réfutées depuis longtemps et depuis longtemps devenues profondément provinciales et nihilistes. En privant le romancier de son droit au monde entier, de la participation à la vie de toute l'humanité, à la recherche de la vérité, à l'amour pour l'homme, ne croyez-vous pas, Claude Simon, que 
vous contribuez au changement de l'écrivain en un petit et insignifiant employé immergé dans son propre vide? Vous avez peur qu'en se jetant dans le monde réel, l'art ne se perde. Mais si l'art perd ce monde, il va se perdre lui-même.

Les idées de Ermilov, si préhistoriques et profondément provinciales qu'elles puissent apparaître au lecteur occidental, sont pourtant compréhensibles et saines dans le contexte général de la littérature russo-soviétique, et nous allons le voir plus bas à l'occasion de l'analyse des opinions de Léonide Andreïev, le critique soviétique le plus perspicace de l'œuvre simonienne. Pour celui qui est né en URSS, il ne faut qu'un peu d'honnêteté intellectuelle pour admettre à quel point était surréaliste, parfois même franchement faux ${ }^{13}$ (et l'est resté jusqu’à la fin) le mythe russe créé par Simon.

Tamara Balachova, docteur ès lettres, directrice de recherches à L'Institut de la littérature mondiale de Moscou dans son livre Le Roman français des années 60. Traditions et recherches ${ }^{14}$ (1965) joue sur l'opposition, habituelle pour la critique soviétique d'alors, entre les bons nouveaux romanciers (le plus souvent c'était Nathalie Sarraute mais, dans ce cas, c'est Michel Butor) et les mauvais, et cela, dès les premières pages: "Une école du "nouveau roman ${ }^{15}$ " est formée qui a mis à nu de la manière la plus radicale le conflit entre des recherches faussement novatrices et de vraies recherches ${ }^{16}$.» T. Balachova consacre à Butor un chapitre à part de son ouvrage, et ne mentionne qu'une fois Claude Simon:

Boue, ennui, hennissement des chevaux, altercations plutôt grossières des anciens soldats [c'est-à-dire des prisonniers], et dans l'imagination - salons luxueux, dames élégantes, messieurs affables, mariage d'un oncle riche, puis - la nuit qui unit à la jeune mariée le héros remplaçant le vieillard, et de nouveau - splendeur du bal et boue des baraques de prisonniers... Tel est l'an 1940 vu par l'auteur des Routes des Flandres, 1961 (sic). Inutile d'essayer de distinguer ce qui avait lieu de ce qui n'avait pas lieu. Importe à l'écrivain l'impression seule - ennui, boue, caractère illusoire du bonheur ${ }^{17}$.

Comme on peut le voir, T. Balachova ajoute aux reproches des critiques précédents encore un grief, celui de l'impressionnisme, ce qui est plutôt étonnant de la part de l'une des plus grandes spécialistes soviétiques de la poésie française du $\mathrm{XX}^{\mathrm{e}}$ siècle.

13. De ce point de vue, les déformations les plus criantes imposées par Simon à la problématique russo-soviétique sont dans L'Invitation, Album d'un amateur et, surtout, dans Le Jardin des Plantes. Les méandres et les causes profondes de cette vision déformante demanderaient un examen détaillé.

14. Tamara Balachova, Le Roman français des années 60. Traditions et recherches, Moscou, "École supérieure ", 1965.

15. Variante de la graphie traditionnelle pour la critique soviétique d'alors (et même, hélas, à présent) où lettre minuscule et guillemets sont appelés à montrer l'attitude négative envers cette étiquette et ce phénomène. Il y avait pire encore: une école du soi-disant "nouveau roman".

16. Ibid., p. 4.

17. Ibid., p. 94 . 
Maurice Vaxmacher, traducteur réputé de la littérature (et surtout de la poésie) française contemporaine (d'Apollinaire, d'Aragon, d'Éluard et d'autres...), dans son ouvrage Littérature française de nos jours ${ }^{18}$ (1967), a mêlé la forme poétique de ses traductions avec la langue et le style de bois de la pire propagande soviétique:

Pour N. Sarraute, A. Robbe-Grillet, M. Butor, C. Simon, C. Mauriac [...] le vieux roman a fait son temps - et avant tout parce qu'il est vieux. Il est selon eux mauvais parce qu'il est vieux. L'art doit se renouveler sans cesse. Se renouveler pour un renouvellement. Si quelque chose a déjà eu lieu dans l'art ce "quelque chose " ne doit pas être utilisé dans le roman moderne. Le nouveau est déjà bon parce qu'il est nouveau. Le vieux roman peignait une réalité visible pour tous, mais il y a une réalité invisible, les pulsions de la subconscience, et les représenter signifie donc représenter une vraie réalité, seule digne d'être l'objet de l'art. Mais il n'est possible de le faire qu'en passant à d'autres formes de l'écriture nouvelles qui sont meilleures déjà et avant tout parce qu'elles sont nouvelles, etc. ${ }^{19}$.

Cette tentative qui consiste à remplacer - plus ou moins consciemment, habilement et cyniquement - l'analyse concrète par un patchwork de clichés et de sentences sans appel pour des délits le plus souvent imaginés est, malheureusement, très répandue dans la critique soviétique et postsoviétique. Attribuer aux artistes novateurs ses propres idées (en tout cas, pas les leurs!) pour les mâcher, remâcher, avaler ou recracher enfin - cela reste la voie la plus commune pour une critique faite dans sa majeure partie, je le rappelle, par des spécialistes respectés et non par des journalistes omnivores, pressés et superficiels.

Leonide Eremeev, docteur ès lettres, a publié à Kiev en 1974 l'unique ouvrage monographique soviétique sur Le "nouveau roman " en France ${ }^{20}$ où il mentionne dix fois Simon et analyse sur quatre pages la version anglaise (en lisant son petit livre, on suppose que le français n'est pas sa première et principale langue étrangère) de l'article de Simon de 1964 «Whom Sartre writes for? " Mais pour nous, les dissertations de Eremeev sont intéressantes du fait qu'ici l'étiquette de formaliste, qui va poursuivre longtemps l'imagination de Simon, apparaît pour la première fois.

Il commence par une tournure impersonnelle pateline:

Il est connu que la prose raffinée des nouveaux romanciers ne trouve ses lecteurs que dans le milieu restreint des intellectuels et des universitaires. La cause de l'insuccès [du Nouveau Roman] est le formalisme. C. Simon pose pour l'écrivain des objectifs formels, et, en s'appuyant sur Picasso, cherche la confirmation de la justesse de sa conception de la création. Simon ignore consciemment les liens indissolubles entre la forme et le contenu [dans la réception d'une œuvre d'art], mais au fil de ses raisonnements, il établit arbitrairement la subordination de l'impression produite par l'œuvre d'art à la psychologie de la création. Est douteux l'éloge d'un admirateur qui voit un mérite de l'œuvre d'art dans le fait qu'il "n’y est rien resté du projet initial ». Le projet initial de Picasso est très bien vu dans la

18. Maurice Vaxmacher, Littérature française de nos jours, Moscou, « Houdojestvennaya literatoura ", 1967.

19. Ibid., p. 130-131.

20. Leonide Eremeev, Le "Nouveau Roman » en France, " Naoukova Doumka », Kiev, 1974. 
mesure où Guernica a été destiné à l'Exposition universelle de 1937 à Paris. Devant l'entrée du pavillon d'Espagne, le panneau rappelait la menace fasciste, faisait naître l'indignation contre la guerre. Et ce n'est que lorsqu'il s'est trouvé dans un musée de New York qu'il est devenu l'objet des discussions formelles des spécialistes d'art ${ }^{21}$.

Il est important de savoir que dans les années 70 (et dans les années 80 encore plus) la lutte acharnée contre le formalisme était révolue depuis longtemps. Les travaux de Tynianov, de Chklovski et de tous les autres sont revenus sur la scène critique, et quoique le courant formaliste soit resté non perspectif du point de vue de la critique officielle, il était tout de même toléré, étudié et continué, notamment par l'Ecole sémiotique de Moscou et de Tartou (Y. Lotmann et les autres). C'est pourquoi le test formaliste de Simon (Dites-moi ce que vous pensez des formalistes et je vous dirai à quel point vous êtes un écrivain officiel et acheté par le KGB) qu'il a pratiqué plus d'une fois en URSS me rappelle l'histoire avec Lev Tolstoï qui, à sa première visite en Allemagne pendant la période de sa passion pour la pédagogie, a parlé à tous et à toutes de Berthold Auerbach ${ }^{22}$ et était stupéfait par l'ignorance quasi totale des Allemands sur l'homme qui est " l'esprit de l'humanité » et dont il pensait qu' « il n'y avait rien au-dessus ».

\section{POINTS FAIBLES ET POINTS FORTS D'UNE APPROCHE SOVIÉTICO- RUSSE DE L'CEUVRE}

Maintenant laissons le développement chronologique de notre panorama pour voir, à partir de l'exemple de Léonide Andreïev, presque tous les côtés faibles et forts des approches de la critique soviétique et russe de l'œuvre simonienne. Ce philologue et professeur célèbre a travaillé cinquante ans à l'université Lomonossov de Moscou dont il a dirigé le département de littérature étrangère pendant 25 ans. Et pendant ces 25 ans, il a écrit plus d'une fois sur Claude Simon. Fin connaisseur de la littérature française, il a lu ma thèse sur Simon en 2001 et l'a approuvée (en dépit du fait que j’y ai critiqué sa fidélité aux appréciations de Simon dépassées depuis longtemps) juste avant sa mort.

Dans son livre Littérature contemporaine de la France. Les années $60^{23}$ (1977), il analyse plutôt en détail les caractéristiques (cinq pages) de l'œuvre de Simon à partir du matériau tiré essentiellement de La Route des Flandres. Le critique parle du naturalisme du Nouveau Roman et de celui des romans de Simon notamment comme de l'expression d'un refus d'évaluer la réalité reconstituée avec méticulosité, attitude interprétée dans la période précédente comme un refus de regarder la réalité en face. Il écrit ainsi :

21. Ibid., p. 210-212.

22. Berthold Auerbach (1812-1882), écrivain allemand d'origine juive, analyste de la pensée de Spinoza, auteur d'ouvrages de fiction, nourri du folklore de l'Allemagne du Sud.

23. Léonide Andreïev, Littérature contemporaine de la France. Les années 60, Moscou, Les éditions de l'université Lomonossov, 1977. 
dans son roman le plus connu La Route des Flandres qui termine les années 50, c'est l'écrivain lui-même qui, sans cesse, invite le lecteur à se tourner vers la réalité, vers une réalité - incontestable et cauchemardesque - de la guerre. Une restitution, scrupuleuse, d'un naturalisme méticuleux, des "fragments ", propre à la manière de Simon, n’a pas empêché dans ce cas-là la création du tableau de la défaite, de la débâcle, du chaos qui s'établit ${ }^{24}$.

Cette idée dialectique de la défense et de l'illustration des réalités référentielles dans la poétique et l'écriture de Simon qui apparaissent peut-être malgré ses propres déclarations hyper-formalistes de toujours, Andreïev va la détailler, l'enrichir, la peaufiner durant des années.

Le tableau du chaos universel qui surgit pendant la lecture du roman est, bien sûr, moderniste. Et toutefois l'écrivain, encore et encore, nous fait revenir sur la route des Flandres, dans les baraques des prisonniers, vers cette réalité inébranlable et concrète de la Seconde Guerre mondiale dont Claude Simon a été un des soldats ${ }^{25}$.

En 1983, les éditions soviétiques les plus prestigieuses n'éditant en règle générale que les classiques de la littérature mondiale « Houdojestvennaya literatoura " (Les Belles Lettres) entreprennent un projet unique, non seulement pour la pratique éditoriale soviétique, mais pour toute l'histoire du Nouveau Roman. Sous la même couverture, avec une grande préface et des notices biographiques de Andreïev sont publiées les traductions de quatre nouveaux romans: La Modification de Butor, Dans le labyrinthe de Robbe-Grillet, La Route des Flandres de Simon (dans une bonne traduction de Evguenia Baboun où, tout de même, sont parfois adoucis des passages érotiques et... antireligieux, ce qui est étonnant pour un pays d'athéisme triomphant), et Vous les entendez? de Sarraute ${ }^{26}$. Le tirage était fantastiquement élevé (50 000 exemplaires) mais on disait tout bas à l'époque que c'était un geste avant tout politique, et qu'une partie écrasante du tirage avait été détruite au lieu d'être proposé dans les librairies, ce que l'impossibilité d'acquérir ce livre, à l'époque, et encore aujourd'hui, prouve indirectement.

Ce n'est plus le désir (ou le devoir) de la "polémique " (politique ou idéologique) dans le genre d'un Ermilov qui guide Andreïev. Ce docteur ès lettres et auteur de la série de cours sur le "nouveau roman ", à la faculté des lettres de l'Université Lomonosov de Moscou, se fixe le but de préparer le lecteur soviétique à la rencontre si longtemps attendue avec les hérétiques de la littérature française, aimée plus que toutes les autres par notre public, « raffiné et averti " autant que " large et divers".

24. Ibid., p. 656 .

25. Ibid., p. 66.

26. Michel Butor La Modification, Alain Robbe-Grillet, Dans le labyrinthe, Claude Simon La Route des Flandres, Nathalie Sarraute, Vous les entendez? introduction et notice L. Andreïev, Moscou, " Houdojestvennaya literatoura ", 1983. Le roman de Simon se trouve aux pages 353-572. 
Quatre romans français dont notre lecteur va faire connaissance appartiennent à l'école littéraire du "nouveau roman » [...]. On pourrait dire qu’aucun phénomène de la littérature étrangère du milieu du $\mathrm{xx}^{\mathrm{e}}$ siècle n'a provoqué des débats d'une telle ampleur que ceux liés au "nouveau roman ». [...] À l'heure actuelle les discussions se sont calmées, le «nouveau roman " est entré dans l'histoire, devenu un fait indiscutable de l'histoire littéraire. [...] Le " nouveau roman » est plus complexe, plus considérable que ne le manifeste l'opinion courante sur lui. La lecture des nouveaux romans est une affaire inaccoutumée pour un lecteur ordinaire, relativement peu de lecteurs ont fait à cette époque (dans les années $50-\mathrm{AV}$ ) sa connaissance. [...] Le lecteur de ce livre sera sûrement frappé par une nouveauté voyante de ces romans [...]. Cependant, le «nouveau roman » ce n'est pas seulement de nouveaux procédés d'écriture mais aussi et surtout une conception du monde réalisée à travers le genre romanesque, et il faut le juger non selon ce qu’il n’a pas ou a nié mais selon ce qu'il est devenu en réalité ${ }^{27}$.

Dans cet article, Andreïev nuance et explique sa réflexion sur le "naturalisme » du Nouveau Roman qui, selon toute apparence, lui a été si chère qu'il y revient même dans ses écrits postsoviétiques ${ }^{28}$. Cette constance montre d'ailleurs le caractère sincère de cet étonnement d'un critique bienveillant pour Simon et le groupe auquel il est apparenté, mais dérouté par ce "refus " des Nouveaux Romanciers de choisir - en les triant - les faits de la réalité, une stratégie qui n'était en vérité qu'un changement de façon de les trier - en les recréant:

À en juger par les déclarations de principe des "nouveaux romanciers ", le " nouveau roman " pourrait apparaitre comme une variante du naturalisme moderne (ne décrivant que des " miettes ", que des fragments de la réalité sans même tenter d'en comprendre la substance). Mais à la différence du naturalisme classique, dans le "nouveau roman ", c'est la conscience individuelle qui passe au premier plan. [...] Le " nouveau roman " n'a pas fondé sa propre recherche sur l'absence de la signification, bien que cette recherche, ce désir de créer un roman "à partir de la poussière ${ }^{29}$ " détermine beaucoup de choses principales dans le «nouveau roman » et dans son évolution ${ }^{30}$.

Il est plus facile de comprendre le contentement, et même la joie, exprimés dans cette dernière phrase sur l'échec du programme initial de "l'association de malfaiteurs" (N. Sarraute) en prenant en considération le côté pathéticodidactique de la littérature et de la critique russo-soviétique ${ }^{31}$. On conçoit,

27. Ibid., p. 3-5.

28. Il y reviendra, par exemple, dans son article de 2000: «Dans un courant indivisible sont fondus tous les temps grammaticaux, tous les événements, toutes les "choses" qui se répètent et se métamorphosent. [...] les cadres des normes grammaticales sont trop étroits pour l'hypernaturalisme de Simon, pour son penchant pathologique pour le superflu des détails, pour des précisions interminables produisant des parenthèses innombrables $[\ldots]$, pour des descriptions incroyablement proliférantes qui ont un effet visuel et pittoresque incontestable ", dans Henri Bergson, Le Rire, Jean-Paul Sartre, La Nausée, Claude Simon, La Route des Flandres, Bibliothèque des Prix Nobel, Moscou, " Panorama », 2000, p. 601-602.

29. Allusion à la formule célèbre de Robbe-Grillet: « à partir de rien, de la poussière » dans Pour un nouveau roman, Minuit, 1963, p. 42.

30. L. Andreïev, op. cit., p. 6-7.

31. Pensons à ce passage, très connu chez nous, qui serait probablement digne, selon Simon, de Jdanov et qui appartient pourtant à son cher Dostoïevski notant pendant le travail sur L'Idiot dans ses Carnets: «Dans la poésie, il faut avoir de l'ardeur, votre propre idée et - absolument - un doigt qui indique, qui 
dans cette mesure, la logique du traitement imposé au Nouveau Roman et à Simon par la critique - même bienveillante - de mon pays:

Chez Claude Simon le courant de conscience est sur l'avant-scène entraînant et engloutissant tout [...]. Son rythme subjectif détruit toutes les normes, même de langue et de syntaxe [...]. Tout tourne dans un tourbillon ininterrompu comme dans le labyrinthe d'Alain Robbe-Grillet. Et pourtant il est possible de déduire du roman La Route des Flandres une histoire bien définie. [...] Donc, dans le roman de Simon n'y a-t-il pas tout de même tout un système des personnages et leur histoire? Mais tout cela apparaît dans le courant de conscience qui ne sert pas à définir un personnage concret mais son for intérieur ${ }^{32}$.

Des qualifications jusqu'alors inouïes dans la critique soviétique apparaissent:

Avec une force d'ensorceleur, C. Simon reproduit par le texte même du livre le rythme d'un mouvement incessant, d'un chevauchement interminable qui remonte vers l'essence de l'être, du courant de la vie. [...]; la mort du capitaine fait écho à la mort de son ancêtre. C'est ainsi que s'affirme le caractère immuable, prédestiné, inconsolable, répétitif des morts, [...] c'est ainsi que se construit l'image d'un abîme dévorant tout. Dans cet abîme plonge l'Histoire avec tous ses mythes et légendes. [...] La tragédie tourne à la farce ${ }^{33}$.

Un autre thème puisé dans $\mathrm{La}$ Route des Flandres et pour la première fois pris au sérieux par un critique soviétique est le thème - sacré et sacral dans notre mentalité et relatif à l'inconscient collectif - de la guerre contre Hitler. Andreïev apprécie très haut ce niveau du texte de Simon, ce qui est très important pour les études simoniennes en général car c'est le seul, semble-t-il, des critiques de Simon qui ait eu une expérience analogue à celle du romancier français et même encore plus tragique. Léonide Andreïev est en effet allé au front l'été 1941 comme engagé volontaire et est revenu à l'université en 1945 totalement mutilé et avec des béquilles. Sous sa plume, le thème officieux de l'antimilitarisme devient un argument décisif " pour » Claude Simon en russe:

La guerre est entrée dans l'œuvre de Simon comme un leitmotiv, comme l'élément le plus important de sa perception du monde, grâce à quoi son meilleur roman, La Route des Flandres (1960), peut être à bon droit appelé « antimilitariste ». Toutefois, ses autres romans détruisent le système des déterminants existant objectivement dans la société. La problématique sociale dont Simon a pourtant mesuré toute l'importance, à ce qu'il paraît, à partir de sa propre expérience vient au second plan en cédant la place à ce que l'écrivain croit éternel, inchangeable dans la vie de tout être humain inscrit dans le monde des choses et lui donnant des leçons instructives ${ }^{34}$.

C'est dire que l'accueil critique de Simon chez nous n’a jamais été simplement négatif, et si on critiquait le romancier français, c'était surtout non

attire l'attention, doigt d'un prophète levé passionnément. L'indifférence et la reproduction fidèle de la réalité ne valent rien du tout et, ce qui est encore plus important, ne signifient rien ». Comment ne pas se rappeler ici le fameux mot répété par Simon dans le Discours de Stockholm après "Shakespeare, Barthes " et Robbe-Grillet - non nommé et refoulé ici selon un processus psychanalytique: «Si le monde signifie quelque chose, c'est qu'il ne signifie rien ", E I, p. 898 et 1464 et Cahiers Claude Simon, n 7, p. 224.

32. L. Andreïev, op. cit., p. 13.

33. Ibid., p. 14.

34. Ibid., p. 355. 
pour son formalisme mais pour sa volonté de limiter son talent de visionnaire onirique et on l'appréciait - beaucoup plus que ses collègues - pour cette étoffe riche et tolstoïenne - malgré ses réfutations de l'héritage du moraliste russe - de sentiments, d'observations justes, profondes, paradoxales et impitoyables. Dans les années 60-80 dans la littérature soviétique apparaissent les romans sur la guerre baptisés prose des sous-lieutenants (c'est un peu l'équivalent du grade de brigadier de Simon - mi-soldat, mi-officier). Ces écrivains (Nekrassov, Bykov, Bondarev, Vorobiev, Astafiev) avec leur vérité des tranchées allaient à contre-courant de l'idéologie officielle; et quand Andreïev interprétait La Route des Flandres comme une fresque tragique mais typique, même dans ses " déformations formalistes ", il rapprochait Simon - en dépit de son élitisme - de ce courant vériste de la littérature soviétique en mettant en relief le niveau référentiel de son texte autour duquel les simoniens occidentaux menaient à cette même époque des combats acharnés.

Dans les années suivantes, il est arrivé à Andreïev d'écrire encore sur Simon-dans le manuel de la littérature française pour les étudiantsphilologues (1987), dans le chapitre Anti-drame et antiroman, dans l'ouvrage académique Littérature française. 1945-1990 (1995) mais les dernières citations de lui que je veux présenter sont tirées d'un volume peu banal ${ }^{35}$ où Simon a comme " colocataires " Bergson avec Le Rire et... Sartre avec La Nausée. Dans son article, Andreïev écrit :

Je suis obligé $[\ldots]$ de montrer à Simon sa contradictio in adjecto et de dire sans détours: La Route des Flandres est devenu le livre le plus important non seulement dans l'œuvre de Simon mais aussi dans l'héritage de tout le groupe des " nouveaux romanciers " parce que son auteur permet au lecteur de trouver dans ce texte une certaine "histoire ", l'oblige à y trouver une signification certaine. Tous les procédés élaborés antérieurement par Simon sont là, et pourtant ils y jouent le rôle d'une forme substantielle qui travaille pour le compte de cet effet puissant que ce livre produit. C'est grâce à cela que La Routes des Flandres est l'un des ouvrages les plus « lisibles » de Simon...

qui " contrairement aux postulats du "nouveau roman" [...] permet au lecteur qui ne craint pas les difficultés de déduire tout un système des personnages de ce roman ${ }^{36} »$.

Et voilà la dernière réplique de Andreïev dans sa discussion de plus de trente ans avec et pour Simon:

Qu'est-ce qui fait de ce roman un grand livre, un événement dans la littérature de deuxième moitié du $\mathrm{XX}^{\mathrm{e}}$ siècle? L'art d'un maître remarquable, soit, mais qu'est-ce que cet art? Des soi-

35. Henri Bergson, Le Rire, Jean-Paul Sartre, La Nausée, Claude Simon, La Route des Flandres, « Bibliothèque des Prix Nobel ", Moscou, "Panorama », 2000. Dans cette série sont toujours présents les discours des prix Nobel, c'est pourquoi y est également publié dans la traduction de Galina Béliaéva le Discours de Stockholm de Simon (p. 579-596) - après le roman (p. 351-578) et avant l'article de L. Andreïev "Claude Simon. Un désordre ordonné » (p. 597-605).

36. Ibid., p. 599-600. 
disant procédés du simultanéisme cubiste, de peinture verbale? Sans aucun doute, et toute cette technique a, bien sûr, permis de produire un tableau inoubliable du « désordre » comme étant un état réel de la société à une étape de son Histoire. L'expérience de cette Histoire, l'expérience vécue par Claude Simon lui-même est devenue une composante des plus importantes de l'artiste et non pas restée un monde - extérieur et parallèle - d'une réalité entre guillemets, comme veut le faire croire l'écrivain ${ }^{37}$.

En 1984, les mêmes éditions qui ont préparé l'année précédente le volume collectif du Nouveau Roman publient un ouvrage de Lénina Zonina ${ }^{38}$ s'intitulant Sentiers du Temps. Notes sur la quête des romanciers français (les années 60-70). Dans la dialectique manichéenne de la critique soviétique, le Nouveau Roman passe désormais au royaume de la lumière en laissant sa place du royaume des ténèbres au "nouveau nouveau roman ", ce qui se manifeste déjà dans le titre du chapitre consacré à ces deux courants - "Recherches et impasses » dont la première partie (p. 59-131) traite du Nouveau Roman et la deuxième, au titre éloquent de " Mise à mort du roman " (p. 131-149) évoque l'action de Sollers et de Ricardou. Les Fab Four du Nouveau Roman sont traités ici dans l'ordre avec les nombres de pages suivants: Sarraute, 16 pages, Butor, 16 pages, Simon, 14 pages $^{39}$, Robbe-Grillet, 23 pages.

Dans ses impressions et appréciations, L. Zonina, tout comme Andreïev, parle du côté ésotérique, hypnotique de la poétique simonienne, ce qui est extraordinaire pour la critique soviétique:

En lisant Claude Simon, il est indispensable de s'adonner au mouvement ensorceleur d'une avalanche épaisse et scintillante du texte. Ce sont une épaisseur et un scintillement de la texture textuelle qui remplissent cette texture de significations plurielles, coulant en s'entremêlant. La phrase de Claude Simon s'étire interminablement, en échafaudant et en surajoutant épithètes, comparaisons, parenthèses. Le texte respire, bat comme un cœur, se ramifie. Une impression naît que l'écrivain cherche, trébuche et trouve enfin un sens, caché de lui-même, à travers ce qu'il écrit. L'interaction de la fable et de la narration est renversée - ce n'est plus la logique d'un sujet, d'un caractère, d'une situation qui cherche à s'exprimer dans un verbe adéquat mais ce verbe même - polyphonique, à plusieurs sens et assonances - qui emporte impérieusement le romancier, et il lutte contre [...] cette insoumission qui lui résiste, qu'il ne peut pas tenir en bride, même en la serrant, même en tâtonnant au long du sentier de la signification - tortillant, capricieux, couvert d'une herbe épaisse. Le texte est la trace de cette lutte ${ }^{40}$.

37. Ibid., p. 603.

38. Lénina Zonina, Sentiers du Temps. Notes sur la quête des romanciers français (les années 60-70), Moscou, "Houdojestvennaya literatoura ", 1984. Traductrice, critique, interprète personnelle de Sartre, et ayant vécu une passion avec lui pendant sept ans, Lénina Zonina connue en France sous le prénom de Lena, a traduit Les Mots (dédiés à Madame Z) et Les Belles Images. Le titre de son livre d'essais joue sur la coïncidence en russe des formes des mots sentiers et tropes.

39. Le même texte est reproduit dans l'ouvrage collectif déjà mentionné, Littérature française. 1945-1990 (1995), et présenté comme un chapitre consacré à Claude Simon où sa tonalité lyrique, parfois un peu pompeuse - normale dans un livre d'essais ou dans des notes, selon le sous-titre donné par l'écrivaine à son ouvrage initial - sonne un peu faux sur le fond académique général.

40. Ibid., p. 94 . 
J'ai déjà mentionné des continuateurs de la cause du formalisme dans les années 60-80 qui admiraient non seulement l'OPOYAZ ${ }^{41}$ (auto-appellation des formalistes) des années 20-30, mais le structuralisme français dans ses formes les plus radicales et rigides, ce qui les a amenés (avec, de plus, l'extase commune de l'intelligentsia face au progrès des sciences naturelles) à l'adoration sans mesure des structures cristallisées, des schémas et des diagrammes de toutes sortes, de la langue des machines qui allait s'établir en maître d'un jour à l'autre... On peut sentir tout cela dans le fragment suivant qui prouve qu'aucun rideau de fer n'a pu arrêter la marche triomphale de la doxa ricardolienne à travers les cerveaux de ses critiques mêmes:

Le texte de Simon est une installation qui absorbe toute l'information du monde et la traite de manière à obtenir à la sortie, à côté du produit de ce traitement qu'est le roman, le principe de son activité conçu pendant ce remaniement. Ce comment le roman se construit-il est son sujet même. La construction même de la phrase imite et modèle le processus du " traitement" de l'information dans et par le texte ${ }^{42}$.

\section{AUTOUR DU PRIX NOBEL}

L'article auquel je voudrais maintenant m'attacher porte un titre en apparence neutre "Reconnaissance ou épitaphe? Au sujet d'un prix international ». Il est publié dans le magazine littéraire Inostrannaya literatoura (Littérature étrangère) $\mathrm{n}^{\circ} 11,1986^{43}$. Son auteur est Alexandre Stroev ${ }^{44}$, spécialiste de littérature comparée. Voilà ce que A. Stroev m'écrit en réponse à une lettre du 28 août 2011, où je sollicitais de sa part un éclaircissement sur les circonstances de la rédaction de cet article:

J'ai écrit cet article sur commande (mais Aitmatov n'était pas encore rédacteur en chef d'Inostrannaya literatoura ${ }^{45}$ ) en réaction à un événement. On ne m’a rien imposé (si je me

41. OPOYAZ, acronyme d'Общество изучения Поэтического Языка, littéralement Société pour l'étude du Langage Poétique, le cercle des chercheurs, critiques et linguistes qui a été créé en 1916 et a existé jusqu'au milieu des années 30. Ses représentants les plus brillants et originaux sont Chklovski, Eichenbaum, Tynianov, Tomachevski, Jacobson.

42. Ibid., p. 95.

43. Alexandre Stroev, « Reconnaissance ou épitaphe? Au sujet d'un prix international », Littérature étrangère, $n^{\circ} 11,1986$, p. 214-218. Ce numéro est envoyé à l'imprimerie le 22 août et signé pour l'impression le 15 octobre 1986. Donc, l'article est écrit avant l'arrivée de Simon en URSS et publié pendant son séjour, entre le 10 et le 21 octobre, à la suite de l'invitation d'Aïtmatov qu'il a d'abord déclinée. Faisons l'hypothèse que ce refus initial de Simon explique en partie certains traits de l'article.

44. Dans l'ouvrage académique, Littérature française. 1945-1990, Moscou, "Naslédié ", 1995 (préparé dans ses grandes lignes pendant la même période que l'article examiné), Stroev est l'auteur des chapitres sur le " nouveau roman ", Robbe-Grillet, Sarraute, Butor (p. 394-447).

45. Il l'était de 1988 à 1990, et c'est à cette période qu'on a projeté d'y publier L'Invitation dans la traduction de Marina Arias-Vihil et Les Géorgiques. Ces projets ont été délaissés après le départ d'Aïtmatov à Luxembourg où il a été, jusqu'à sa mort en 2008, ambassadeur de l'URSS, de la Russie et du Kirghizstan auprès du Benelux. 
rappelle bien, on m'a demandé d'évoquer la liste des prix Nobel de littérature), j'ai écrit ce que je voulais (avec, évidemment, une autocensure, obligatoire en 1986). Tout ça n’avait rien de commun avec l'invitation de Simon en Russie.

Cet article est, selon moi, frappant de plusieurs points de vue. D'un côté, il est composé avec une perfection et un professionnalisme rares, même pour le magazine du niveau d'Inostrannaya literatoura: clair, logique, examinant sans bavardage habituel et rituel, le problème annoncé, riche en citations, observations, noms, faits et titres évoqués. D’un autre côté, sa lecture laisse un arrière-goût trouble et troublant. Et à la relecture, cette impression se précise, s'éclaircit et se résume à ceci: son auteur n'aime pas Claude Simon ${ }^{46}$. Sans doute n'est-ce pas un problème en soi, il y a tant de critiques et de chercheurs qui trouvent Simon ennuyeux! Mais ici, il ne s'agit pas d'un Jacques Brenner avec sa haine zoologique (comme cela s'appelait en URSS) contre le Nouveau Roman mais d'un auteur qui, tout en déclarant son objectivité et en évitant de répéter les vieilles âneries soviétiques, essaie d'amener son lecteur à partager ses opinions qui sont loin d'être objectives ou, du moins, neutres.

Cette stratégie critique envers Simon, bien connue en France (on peut penser, par exemple, à l'anecdote la plus "étrange " (M. Calle-Gruber), celle de la «mystification » (Simon) de Robbe-Grillet à Paris Match en octobre 1985 ${ }^{47}$ ) mais inédite jusqu'alors en URSS, me rappelle la première épigraphe de la nouvelle célèbre de Pouchkine omise par Mérimée dans sa traduction: " "Dame de pique signifie une inimitié cachée". Tiré d'un nouveau livre de prédictions d'avenir ». Réticences, allusions, rumeurs savamment réunies et dosées sont entremêlées à des citations de Simon que le critique ne donne le plus souvent que pour les démentir, nier, tourner en ridicule. À la casuistique condescendante et sans appel de Samariy Vélikovski ${ }^{48}$ de 1963 dans le même magazine, Stroev ajoute de l'ironie, ce qui rend encore plus perceptible la tonalité dominante de son article.

Cette tonalité, ressentie déjà dans le titre et le sous-titre, est donnée dès la première phrase: «L'attribution du prix Nobel de la littérature de 1985

46. Ce que Alexandre Stroev a confirmé dans une lettre qu'il m’a adressée: "Je crois que nos divergences [dans les opinions sur Simon et le Nouveau Roman] s'expliquent facilement: vous aimez les romans de Simon, et moi, à vrai dire, je les trouve ennuyeux, à la différence des livres de Sarraute, de RobbeGrillet et de Butor. "

47. Voir M. Calle-Gruber, op. cit., p. 361. A. B. Duncan, dans sa Notice pour le Discours de Stockholm (E I, p. 1456) parle de Robbe-Grillet " mélangeant le fiel au miel » dans son article pour Libération.

48. Par exemple: «Malgré toutes les variantes de "nouveau roman” créées par chaque participant de l'école, elles coïncident toutes dans l'aspiration à repousser aux arrière-cours de la littérature une personnalité entière, intégrale, en élaborant la formule de la narration "déshumanisée", "pure". [...] Ils n’ont inventé en tout cela rien de nouveau. [...] Comme les néoromanciers français [...] poussent au raffinement extrême la méthodologie puisée dans l'arsenal des sophismes auxquels a recours la pensée d'une classe atteinte d'un mal incurable de caducité, d'une classe quittant l'arène historique, leur laboratoire fonctionne à vide ", S. Velikovski, op. cit., p. 180, 191. 
au prosateur français Claude Simon est devenue un événement hors pair, provoquant une réaction mitigée ". En constatant ensuite l'échec du "nouveau roman " en tant que mouvement d'avant-garde, l'auteur semble donner la parole à Simon: "Mais comment tout de même pourrait-on expliquer le choix de l'Académie suédoise? En effet, comme l'a dit Claude Simon luimême dans son discours du Nobel, [...] d'autres auraient pu aussi obtenir cet honneur. " Ensuite il donne la liste des onze prétendus prétendants au Nobel 1985. En relisant cet article, je ne pouvais pas ne pas penser qu'il était écrit dans l'intention de mettre en valeur deux affirmations: celle-ci, " ont été également discutées les candidatures de Günter Grass (RFA), d'un poète et essayiste mexicain Octavio Paz, et de Tchinguiz Aïtmatov et les chances de ce dernier - à en juger d'après la presse française - étaient réelles " (p. 214), et le dernier alinéa de l'article cité plus bas.

Ensuite l'auteur revient à Simon et donne deux citations du communiqué de presse de l'Académie suédoise, mais seulement pour annoncer que "ces lieux communs disent peu de l'individualité artistique de Claude Simon" et il ajoute, pour sa part, qu'il est "un écrivain remarquable et important». Il s'agissait manifestement de " corriger " les appréciations des académiciens et de changer la "reconnaissance " en " épitaphe ». Les quatre pages qui suivent utilisent le même procédé: une thèse appartenant ou attribuée à Simon - et son érosion à travers des tournures impersonnelles ${ }^{49}$, des incises, des précisions rendant ce qui est vague moins clair encore, des verbes à la forme passive ou avec des pronoms indéfinis et des exclamations rhétoriques.

Ensuite Stroev donne encore quelques citations de Simon auxquelles il apporte la conclusion suivante: «Il y a trente ans les mots d'ordre des «nouveaux romanciers " étaient apparus comme un cri de révolte, à présent ils sont devenus obsolètes. Le renversement des conventions réalistes s'est changé en un dictat apporté par des règles encore plus conventionnelles. Voilà pourquoi si polémique quait été le discours du Nobel de quarante minutes préparé par Claude Simon, il s'est avéré traditionnel»(p. 216). En invoquant un souci d'objectivité, Stroev cite A. Rinaldi qu'il présente comme "un iconoclaste sonnant le tocsin ". Après quoi il feint de donner un droit à réponse à Simon mais dans la forme particulière déjà analysée:

49. À la fin de l'article, Stroev utilise le procédé de la discussion imaginaire de Simon avec un lecteur - anonyme et global (contemporain de celui des années 50, français et soviétique mais en accord total avec Stroev) amené dans la direction voulue à l'aide d'incises allusives et de mises en gardes contre les hypothétiques erreurs idéologiques du romancier français: «Le lecteur peut (et non sans cause) voir ici une contradiction. Mais il importe à Claude Simon avant tout de défendre sa thèse de la "liberté absolue de la création". [...] Claude Simon aime à rappeler qu'il a lutté contre le racisme et le militarisme et qu'il a même été traduit en justice pour "outrage à l'armée française". Et pourtant, l'absence d'une position politique nette peut se changer en conservatisme des opinions, aboutir aux déclarations irresponsables» (p. 217). 
À la plupart de ces accusations, ou plutôt de ces injures, Claude Simon lui-même a déjà répondu dans son discours du Nobel ${ }^{50}$. Mais essayons de comprendre ce qui se cache derrière elles, peut-être contiennent-elles, à notre grand regret, une part de vérité? (p. 215)

Dans les passages suivants, on peut constater la même attirance-répulsion pour la cristallo-informatique textuelle (que nous avons déjà vue chez Zonina) pour laquelle le texte simonien ne sert que d'un champ d'application de plus. En plongeant la méthode de Simon dans les extases produites par les premiers ordinateurs des années 60 sur l'intelligentsia humanitaire (leitmotiv de l'École sémiotique de Moscou-Tartou), Stroev décrit en fait ses propres fantasmes: "Au début, dans les années 50, cette description - savamment usée, apparemment illogique - des événements répétés sans fin était ressentie par des lecteurs et des critiques comme un degré extrême du naturalisme; les définitions telles que " chosisme ", " école du regard " sont apparues. [...] Il est évident que l'abandon exclusif au processus ou de la "génération des textes " peut aboutir ou à Zaoum de Khlebnikov ou à l'ordinatorisation de la littérature "(p. 215, p. 218).

Le dernier paragraphe de l'article est explicite:

Ayant choisi Claude Simon l'Académie suédoise l'a mis formellement sur le même rang que les grands écrivains du xx siècle: T. Mann, W. Faulkner. Mais ce n'est que formellement... Le prix Nobel n'élève pas son possesseur au-dessus de beaucoup d'autres qui n'ont jamais été honorés par lui. (p. 218)

\section{L'ÉPISODE DU FORUM D'ISSYK KOUL: UNE INVITATION ET SES CONSÉQUENCES}

Il nous reste à aborder l'épisode du forum d'Issik Koul (octobre 1986) auquel Simon a été officiellement convié, les résonances que celui-ci a eues dans son œuvre, en particulier à travers L'Invitation et la manière dont ces éléments ont été perçus dans le cadre russe.

Sergueï (ou Serge) Zenkine, docteur ès lettres, un des spécialistes de la littérature française les plus réputés de la Russie actuelle, connu également comme théoricien de la littérature ${ }^{51}$ rend compte de certaines de ses perplexités dans la postface à la première traduction de L'Invitation en russe (en fait, la troisième) faite par Ekaterina Liamina et publiée en $2003^{52}$; il écrit notamment: «on ne peut qu'être intrigué par [...] les passages du texte où [l'auteur] se contredit lui-même, s'embrouille dans ses propres détails». Et parmi les exemples de " contradictions ", il cite les allusions à Marilyne Monroe qui serait tantôt

50. Discours qui ne sera publié en russe qu'en 2000.

51. Pour l'ouvrage académique Littérature française. 1945-1990, S. Zenkine a écrit des articles de référence sur le genre de l'essai, et dans une perspective théorique sur Bataille, Blanchot et Barthes (ibid., p. 797-841).

52. Simon Claude, L'Invitation (trad. en russe par Ekaterina Liamina, avec la réponse de Simon à quelques questions de Pavel Chinsky et la postface de Sergueï Zenkine), Moscou, OGI, 2003. 
« la plus belle fille du monde », tantôt « la poupée de chair », dédoublement qui trouverait un écho dans les allusions à Miller et à l'" acteur anglais ${ }^{53}$ ", ou dans les "versions parallèles et contradictoires " des paroles de l'interprète liées au désir de " l'invité » de photographier le buste de Lénine qui ne sont en vérité que des touches complémentaires au tableau simonien de la théorie de la conspiration des Soviétiques contre un Occidental.

De ces prémisses quelque peu faussées, Zenkine tire la conclusion suivante:

Mais bien sûr, ce n'est qu'un procédé d'écriture [...] qui a été activement développé pour la première fois $[. .$.$] par le "nouveau roman français » dont l'un des principaux représentants$ a justement été Claude Simon [...]. De concert avec ses complices, Michel Butor ou Alain Robbe-Grillet, il a créé la technique de l'écriture romanesque immobilisée où la narration s'enlise dans la description. Dans des phrases adimensionnelles, extrêmement compliquées, et qui se superposent [...] ne sont possibles ni un acte audacieux ni une parole responsable et réfléchie d'un quelconque personnage. Il est étonnant de voir comment cette technique du « nouveau roman ", inventée pour des sujets et des visées autres, a coïncidé avec la réalité de l'Union Soviétique - ou au moins avec cette image effrayante, surprenante et impénétrable de l'empire soviétique, la seule du reste que pouvait se faire un voyageur étranger ignorant la langue locale et soigneusement isolé des contacts avec la population indigène ${ }^{54}$.

Serge Zenkine développe cette idée dans le passage suivant, très typique de la pensée libérale postsoviétique sans remarquer le caractère contradictoire de son interprétation de la poétique simonienne formulée par lui d'une façon assez éloignée de la vraie problématique de Simon:

La Russie et l'Union Soviétique ont été déjà décrites comme le règne de l'uniformité inhumaine et de la suppression des libertés individuelles par d'autres visiteurs connus et critiques - [...] par Custine, [...] par Gide. Mais Claude Simon est le premier (le dernier?) à utiliser à cette fin une technique narrative particulière qui change le monde entier en monde immobilisé du cauchemar. Paradoxalement, cette conformité - si parfaite esthétiquement, tout à fait adéquate - de la « forme » artistique à « l'objet » historique est apparue dans le moment même où le colosse inerte de l'empire soviétique s'est tout de même mis en mouvement et a commencé à faire demi-tour, tout en tombant en ruines. [...] Cette présentation de l'empire soviétique immobile à l'aide du style immobiliste du " nouveau roman » n'a pas bien sûr eu d'influence sur les événements, mais de façon presque mystique, est devenue la prédiction de son explosion imminente ${ }^{55}$.

Le personnage suivant n'est pas chercheur ou critique proprement dit. Natalia Popova est écrivaine, éditrice, interprète professionnelle et elle a accompagné plusieurs célébrités dans leurs pérégrinations à travers l'URSS,

53. En réalité, ces effets de dédoublement sont imputables à des erreurs de traduction. L'auteur de la postface n’a pas non plus évité une confusion: « Dans la vieille danseuse [...], on reconnaît facilement Galina Oulanova ", ibid., p. 131. Or, celle-ci, née en 1909 a terminé sa carrière en 1960, beaucoup plus tôt que Maïa Plissetskaïa (née en 1925) dont il est question. S. Zenkine a dû être trompé - comme tant d'autres - par la description caricaturale simonienne de la vieille danseuse. En dépit des fantasmes de Simon, ces deux étoiles ont d'ailleurs gardé jusqu'à la fin une forme physique et artistique magnifique.

54. Ibid., p. 133.

55. Ibid., p. 135. 
notamment Claude Simon en $1986^{56}$. Dans son livre Rêves d'autrefois, elle a publié un essai, Tchinguiz Aïtmatov, Issyk-Koul et Claude Simon ${ }^{57}$. Les passages que je vais citer se passent presque de commentaire, et leur violence émotionnelle peut être d'abord expliquée par une réaction par rapport à ce que Simon a écrit sur "son interprète " dans ses textes.

Natalia Popova est sans doute aussi indignée par cette transformation caricaturale, presque cauchemardesque que Simon fait subir à la réalité de l'Union Soviétique, et en tant que personne ayant vécu dans ce pays et dans ce temps, je ne peux que concevoir cette indignation. L'Union Soviétique brejnevienne n'était pas l'enfer totalitaire (ce que Brodski - un des trois « martyrs " du Jardin des Plantes - comprenait très bien), et l'URSS gorbatchévienne l'était encore moins. Presque tout ce que Simon écrit sur le thème russe et soviétique dans ce roman ne correspond pas à la réalité perçue côté russe et les excès de l'ironie simonienne sont, selon moi, la cause principale de la violence de l'essayiste.

Dans le premier fragment N. Popova montre ce qui se passait dans les coulisses de l'invitation de Simon en URSS:

En 1986, quelqu'un de l'entourage de Gorbatchev a donné un sage conseil: celui d'inviter chez nous les intellectuels de pays divers pour briser la glace de la distance et de la méfiance traditionnelles envers l'URSS. Pour voiler les motifs véritables (politiques) de cette action, il était décidé que ce ne serait pas à l'invitation du secrétaire général du PCUS mais à celle d'un écrivain soviétique connu dans le monde. Et l'opinion publique étrangère devait croire que Evtouchenko ou Voznessenski avaient décidé sans rime ni raison de faire preuve d'une hospitalité de tzars et d'inviter des politiciens, des philosophes, des écrivains et des peintres connus du monde entier, et tout cela pour s'asseoir en cercle et débattre dans l'intimité du destin de notre planète ${ }^{58}$.

Ensuite, N. Popova décrit ce voyage jour après jour et s'il importe de la citer un peu longuement, c'est que ce descriptif (peu bienveillant) donne la mesure du malentendu qui s'est installé très vite entre Simon et ses hôtes:

Notre premier jour ensemble s'est passé tout à fait paisiblement et de façon heureuse. Nous avons visité le musée de Kuskovo ${ }^{59},[\ldots]$ goûté la cuisine russe, et sur sa demande, j’ai ramené Claude Simon sur la Place Rouge pour qu'il puisse satisfaire son désir le plus ardent: admirer la relève de la garde devant le mausolée de Lénine ${ }^{60}$. Il faisait très froid,

56. Le Jardin des Plantes fait apparaître, à plusieurs reprises, N. Popova (ou plutôt l'image simonienne généralisée de la femme soviétique car il décrit son interprète de 1984 dans des termes identiques, et son portrait collectif des interprètes dans L'Invitation ne se distingue guère des esquisses ci-dessous) comme: " la femme au visage malingre, [...] grisâtre, fatigué et sans âge, [...] fané », $E I$, p. 936, 1137, 1138.

57. Natalia Popova, Rêves d'autrefois, Moscou, « Kstati », 2005, p. 283-308.

58. Ibid., p. 284-285.

59. Ancienne propriété de la famille aristocratique de Chérémétieff à Moscou. Musée de céramique et de l'art russe du XVIII ${ }^{e}$ siècle.

60. De toute évidence, Simon le faisait à l'occasion de chaque voyage à Moscou. Ses photos de la Place Rouge dans Album d'un amateur sont faites pendant son voyage de 1984, et dans la nouvelle, Babel, relatant de façon presque documentaire plusieurs circonstances de son séjour de 1937, Simon, descendu 
Claude Simon était transi de froid mais il observait, avec un enthousiasme enfantin, sans remarquer qu'il avait le nez humide nos braves petits soldats marcher à pas mesurés vers le “poste numéro 1”. (p. 286)

Ensuite N. Popova décrit la première journée à Frounze avec promenade en ville, visite du musée local accompagnée de chants et de danses folkloriques, rencontre avec les écrivains kirghizes, cérémonie de plantation d'arbres par les nouveaux citoyens d'honneur, chasse au faucon « refusée à l'unanimité par les hôtes de marque ", invités le soir chez Aïtmatov.

L'écrivaine décrit ainsi cette visite:

Le prix Nobel que j'avais sous ma tutelle ne se distinguait pas par la sociabilité. Et n'avait pas soif de communiquer avec qui que soit. [...] Petit, chenu, toujours dans le même veston de cuir, Claude Simon se promenait d'un air sauvage à travers l'appartement d'Aïtmatov ${ }^{61}$. [...] Le jour suivant, on nous a conduits dans la montagne, au bord du lac d'Issyk-Koul, où les intellectuels, entourés de beauté fabuleuse, devraient élaborer la nouvelle mentalité planétaire appelée à en finir avec la guerre froide. En dépit du scepticisme qu'on peut avoir envers les grands mots et la rhétorique politique [...] je dois dire que cette idée était remarquable. [...] Claude Simon a été le seul qui n’a jamais quitté son humeur maussade.

L'intervention de l'auteur Nobel est devenue, selon N. Popova, le point critique de ce voyage:

Quand on lui a proposé d'intervenir il m'a dit qu'il lirait son discours du Nobel [...]. Je l'ai feuilleté et ai osé lui dire que son contenu ne s'accordait pas trop au thème des discussions générales. Claude Simon a répondu qu'il avait mis toute sa vie pour formuler ses idées et ne pourrait dire rien d'autre...

Tout en précisant avoir dû s'absenter à l'heure de cette intervention, N. Popova ajoute que ce discours a été jugé par certains "ennuyeux » et « insupportablement long... » À en croire l'essayiste, "rien dans pays - sauf la relève de la garde du mausolée - n'a attiré, [...] [l'] intérêt [du romancier français]. Gens rencontrés, villes, paysages d'une rare beauté, monuments de l'histoire, culture russe et culture kirghize, cathédrales et musées du Kremlin, trésors du "Fond des diamants", art dramatique - tout l'a laissé imperturbablement indifférent ${ }^{62}$ ". Si on reste au niveau des "impressions de voyage " traditionnelles, on ne peut pas ne pas être d'accord avec cette conclusion.

Pour comprendre mieux le traitement imposé par Simon à la réalité soviétique, il n'est pas sans intérêt de lire les pages consacrées à la signature de

dans un hôtel en bordure de la Place Rouge, écrit: « [n]ous traversions la Place Rouge, son immense et aride désert de pierres ». Et plus loin, «Place Rouge! hurla-t-il. Red Square! Plaza Roja! Piazza Rossa! Rote Platz! Abruti! Bon Dieu comment est-ce qu’on dit... Krasnaïa... ", Cahiers Claude Simon, n 7 , 2011, p. 14, p. 15.

61. Un peu plus haut, N. Popova écrit que c'était "une HLM soviétique ordinaire » où deux ou trois appartements étaient transformés en un seul, spécialement pour Aïtmatov.

62. Ibid., p. 287-288, 289, 293-294. 
l'Appel à l'humanité et à l'anecdote sur le saumon et de les comparer avec les passages correspondants du Jardin des Plantes ${ }^{63}$ :

Comme Claude Simon ne voulait pas signer la variante anglaise du document final accepté au Forum, Federico Mayor ${ }^{64}$ s'est offert de le traduire de sa main en français. [...] Il a traduit scrupuleusement, mot à mot, ce texte en français. [...] [Le romancier français] s'est obstiné dans son refus mais Mayor l'a persuadé tout de même de signer au moins la variante française. Pour la première fois les joues pâles de Claude Simon sont devenues rouges...

L'écrivaine précise qu'il fallait faire quelque chose du reste de l'«argent de poche " délivré à l'écrivain français, comme à tous les participants du Forum - argent probablement en roubles et donc inutilisable hors de l'URSS. C'est ici qu'intervient l'anecdote du saumon, achetée par l'interprète, et qui aurait suscité de la part du romancier français, une réaction enjouée, présentée comme rare ${ }^{65}$.

Je ne terminerai toutefois pas sur cette note en donnant maintenant la parole, sur le même sujet, à Svetlana Makurenkova, poète, traductrice, docteur ès lettres, qui a été pendant ce Forum, l'interprète d'Arthur Miller et le témoin perspicace des souffrances mutuelles de Simon et de son entourage. S. Makurenkova a écrit un essai non publié sur Simon Notes et observations d'un témoin oculaire dont elle m'a aimablement permis de citer des fragments.

Cet homme d'une taille plutôt petite avec un regard méfiant et un visage soigneusement rasé était habillé dans une veste de jeune homme et en jeans. Dans son attitude envers le monde - hostile et éveillée - il y avait quelque chose d'enfantin, et il ne pouvait pas cacher cet état d'esprit. Son caractère était hérissé comme ses cheveux courts et blancs, qui témoignaient nettement de son tempérament intransigeant [...]. Dans son ensemble, son allure laissait supposer que même si la vie ne lui offrait pas de circonstances défavorables il allait les inventer...

Et la commentatrice explicite très clairement les raisons du dialogue de sourds qui s'est instauré entre Simon et ses hôtes:

De manière mystérieuse, la réalité de son œuvre littéraire s'est dissoute, et le prix reçu pour cette œuvre littéraire est passé au premier plan. [...] Cela rendait sans doute la situation ambiguë: l'œuvre littéraire telle quelle était absente et la récompense de cette œuvre - présente. Il devenait évident que ceux qui avaient invité [l'écrivain français] à ce Forum avaient des visées sur ces honneurs, et Claude Simon, avec son œuvre problématique et difficile, ne les intéressait guère. On peut supposer que Claude Simon lui-même sentait l'ambiguïté de la situation et tentait de toutes ses forces de garder son contrôle sur elle.

63. E I, p. 975, p. 998-999 (pour la scène avec la signature) et p. 1138-1139, 1141-1142 (pour la scène avec le saumon).

64. Federico Mayor Zaragoza (né en 1934 à Barcelone) diplomate et poète espagnol, sera aussi directeur général de l'Unesco (1987-1999).

65. Plus intéressant sans doute pour les admirateurs d'Histoire, N. Popova note à ce propos le caractère économe de l'écrivain français en précisant: «Sa seule petite dépense, c'était des cartes postales innombrables avec des vues locales qu'il envoyait par liasses dans des pays différents de chaque hôtel ou ville où il voyait une boîte postale. " 
Et S. Makurenkova poursuit en ces termes:

cela lui garantissait la liberté certes, mais une liberté d'un genre particulier dont il est devenu l'otage, et le texte de son essai L'Invitation en est le témoignage. Claude Simon s'est trouvé dans une situation de mutisme. [...] Son journal de voyage est un récit d'un genre à part qui est né de cette situation compliquée. Sa particularité consiste dans le fait que tout ce que l'auteur voyait était interprété dans et selon son paradigme de pensée. La voix de la réalité, dans notre cas - celle de la langue russe - n'était pas autorisée à y entrer.

La conclusion de Svetlana Makurenkova n'est pas moins intéressante:

Sans toucher aux profondeurs de la poétique du "nouveau roman », il faut remarquer qu'une pareille dualité engendre la fantasmagorie où la création de la réalité artistique se laisse complètement recouvrir par l'imagination de l'auteur, nullement gênée par la voix de la réalité comme telle. L'Invitation est écrite, si l'on y réfléchit bien, dans une manière qui rappelle vaguement les contes d'Hoffmann. Mais la divergence de l'intention initiale et du sens qui en a découlé est devenue le prix à payer pour la spécificité du genre. Le résultat du refus de Simon de jouer un rôle imposé a fait qu'il a tout de même "joué " ce qu'on attendait de lui, et il l'a fait mieux que n'importe quel autre participant du Forum d'IssykKoul. Lui seul a écrit un livre où il a gravé le moment historique du commencement de la Perestroïka. [...]. Aujourd'hui son livre est un document précieux de ces années agitées.

On peut donc constater que tous les commentateurs russes de l'expérience soviétique de Simon remarquent ce caractère fantasmagorique, très subjectif ou "néoromanesque " de la poétique de L'Invitation, qui ne doit en aucun cas être ignoré pendant l'examen critique de ce texte.

\section{VERS UNE RÉCEPTION PLUS LARGE DE L'CEUVRE DE SIMON EN RUSSIE}

Toutefois, certains spécialistes de littérature française contemporaine n’ont pas voulu s'en tenir aux textes de Simon, centrés ou sur la Seconde Guerre ou sur les voyages russes de l'écrivain Nobel.

Marina Arias-Vihil est collaboratrice de l'Institut de la littérature mondiale de Moscou. Dans l'ouvrage collectif intitulé Littérature étrangère du XX siècle ${ }^{66}$ (2003), elle est l'auteur de l'article sur la littérature française de la deuxième moitié du $\mathrm{xx}^{\mathrm{e}}$ siècle $^{67}$. Sur deux pages, elle donne une vision brève mais assez étoffée des principaux romans de Simon, en construisant ses réflexions autour de la problématique du temps:

Les personnages de L'Herbe [...] n'ont aucune histoire au sens où leur vie est extrêmement médiocre. Néanmoins, dans l'interprétation de Simon, cette matière - vouée à la mort et percée par tous les vents du temps - se met à " chanter ", " renaît " comme un objet d'art. [...] Dans La Route des Flandres [...] apparaît l'image de la matière du temps - dense, sombre et pleine de trous divers. La toile d'araignée de la mémoire tache de la rapiécer mais les fils de chaque homme-araignée ne se croisent qu'approximativement. [...] Le Palace décrit, de toute évidence, la déception de Simon face au marxisme et au désir de transfor-

66. Littérature étrangère du XX' siècle, dir. par Vassili Tolmatchoff, « Academia », 2003.

67. Ibid., p. 391-433. 
mer le monde à l'aide de la violence. Ses sympathies vont aux victimes de l'histoire. [...] Un roman monumental, Les Géorgiques [...] est l'une des œuvres les plus importantes de Simon où l'auteur se tourne de nouveau vers le thème de la collision de l'homme avec le temps. [...] En traitant des relations très complexes entre connaissance, écriture et temps, Simon oppose l'archétype de la terre et des saisons au chaos destructeur de la guerre ${ }^{68}$.

Le chercheur suivant est moi-même. J'ai lu Simon en 1983 parmi les autres "nouveaux romanciers " pendant mes études. Le Nouveau Roman est devenu, d'abord, le thème de ma thèse d'études supérieures en 1985. En 2002, j'ai soutenu la thèse «L'œuvre de Claude Simon dans le contexte de la poétique du Nouveau Roman (sur l'exemple des romans des années 50) » et, en 2011, ai poursuivi par la thèse de doctorat "La poétique du Nouveau Roman " basée, dans sa majeure partie, sur l'examen de l'œuvre simonienne. J'ai publié deux ouvrages en russe consacrés au Nouveau Roman et à Claude Simon et une vingtaine d'articles mentionnés, pour une part d'entre eux, dans la bibliographie. Mon intérêt se porte surtout vers les romans des années 50 relus sur fond de Nouveau Roman et vers les problèmes d'interprétation du texte simonien à travers ses unités de seuil. Le problème théorique que je pose est une tentative pour montrer sur le matériau tiré du Nouveau Roman la possibilité de repérer dans le modèle du texte en miniature qu'est l'ensemble titre-dédicaceépigraphe-incipit-explicit toutes (ou presque toutes) les particularités du texte intégral de l'œuvre. Je suis en train de préparer l'édition critique en russe de L'Herbe et de L'Invitation.

On constate donc que Claude Simon " russe » - aussi bien qu’ « américain ", " espagnol ", " allemand " ou quel encore? - nous prépare plus d'une surprise et plus d'une découverte ce qui prouve une fois de plus le caractère relatif et provisoire de tout notre jugement. L'œuvre et la vie de Simon est - et c'est ce que chaque simonien comprend à un moment donné! - un puits de mystères.

68. Ibid., p. 422-423. 